

\title{
A fast conservative spectral solver for the nonlinear Boltzmann collision operator
}

Irene M. Gamba, Jeffrey R. Haack, and Jingwei Hu

Citation: AIP Conference Proceedings 1628, 1003 (2014); doi: 10.1063/1.4902703

View online: http://dx.doi.org/10.1063/1.4902703

View Table of Contents: http://scitation.aip.org/content/aip/proceeding/aipcp/1628?ver=pdfcov

Published by the AIP Publishing

Articles you may be interested in

High performance computing with a conservative spectral Boltzmann solver

AIP Conf. Proc. 1501, 334 (2012); 10.1063/1.4769536

Conservative deterministic spectral Boltzmann solver near the grazing collisions limit

AIP Conf. Proc. 1501, 326 (2012); 10.1063/1.4769535

Implicitly charge-conserving solver for Boltzmann electrons

Phys. Plasmas 16, 062310 (2009); 10.1063/1.3152288

Structure of the Boltzmann Collision Operator

Phys. Fluids 8, 431 (1965); 10.1063/1.1761242

Eigenfunctions of the Boltzmann Collision Operator

Phys. Fluids 7, 1388 (1964); 10.1063/1.1711389 


\title{
A Fast Conservative Spectral Solver for the Nonlinear Boltzmann Collision Operator
}

\author{
Irene M. Gamba*,†, Jeffrey R. Haack ${ }^{*, \dagger}$ and Jingwei $\mathrm{Hu}^{* *, \dagger}$ \\ *Department of Mathematics, The University of Texas at Austin, 2515 Speedway, Stop C1200 Austin, Texas \\ 78712, USA \\ ${ }^{\dagger}$ ICES, The University of Texas at Austin, 201 E. 24th St., Stop C0200, Austin, TX 78712, USA \\ ** Department of Mathematics, Purdue University, 150 N. University St., West Lafayette, IN 47907, USA
}

\begin{abstract}
We present a conservative spectral method for the fully nonlinear Boltzmann collision operator based on the weighted convolution structure in Fourier space developed by Gamba and Tharkabhushnanam. This method can simulate a broad class of collisions, including both elastic and inelastic collisions as well as angularly dependent cross sections in which grazing collisions play a major role. The extension presented in this paper consists of factorizing the convolution weight on quadrature points by exploiting the symmetric nature of the particle interaction law, which reduces the computational cost and memory requirements of the method to $O\left(M^{2} N^{4} \log N\right)$ from the $O\left(N^{6}\right)$ complexity of the original spectral method, where $N$ is the number of velocity grid points in each velocity dimension and $M$ is the number of quadrature points in the factorization, which can be taken to be much smaller than $N$. We present preliminary numerical results.
\end{abstract}

Keywords: spectral method, Boltzmann equation

PACS: $02.70 . \mathrm{Hm}, 51.10 .+\mathrm{y}$,

\section{INTRODUCTION}

There are many difficulties associated with numerically solving the Boltzmann equation, most notably the dimensionality of the problem and the conservation of the collision invariants. For physically relevant three dimensional applications the distribution function is seven dimensional and the velocity domain is unbounded. In addition, the collision operator is nonlinear and requires evaluation of a five dimensional integral at each point in phase space. The collision operator also locally conserves mass, momentum, and energy, and any approximation must maintain this property to ensure that macroscopic quantities evolve correctly.

Spectral methods are a deterministic approach that compute the collision operator to high accuracy by exploiting its Fourier structure. These methods grew from the analytical works of Bobylev [1] developed for the Boltzmann equation with Maxwell type potential interactions and integrable angular cross section, where the corresponding Fourier transformed equation has a closed form. Spectral approximations for these type of models where first proposed by Pareschi and Perthame [2]. Later Pareschi and Russo [3] applied this work to variable hard potentials by periodizing the problem and its solution and implementing spectral collocation methods. Spectral methods have advantages over Direct Simulation Monte Carlo Methods (DSMC) in many applications, in particular time dependent problems, low Mach number flows, high mean velocity flows, and flows that significantly deviate from equilibrium. In addition, deterministic methods avoid the statistical fluctuations that are typical of particle based methods.

Inspired by the work of Ibragimov and Rjasanow [4], Gamba and Tharkabhushanam [5, 6] observed that the Fourier transformed collision operator takes the form of a weighted convolution and developed a spectral method based on the weak form of the Boltzmann equation that provides a general framework for computing both elastic and inelastic collisions. Macroscopic conservation is enforced by solving a numerical constrained optimization problem that finds the closest distribution function in $L^{2}$ in the computational domain to the output of the collision term that conserves the macroscopic quantities. This optimization problem is the approximation of the projection of the Boltzmann solution to the space of collision invariants associated to the corresponding collision operator [7]. In addition these methods do not impose periodization on the function but rather assume that solution of the underlying problem on the whole phase space is obtained by the use of the Extension Operator in Sobolev spaces. They are also shown in the space homogeneous, hard potential case, to converge to the Maxwellian distribution with the conserved moments corresponding to the endowed collision invariants. Such convergence and error estimates results heavily rely on the

Proceedings of the 29th International Symposium on Rarefied Gas Dynamics AIP Conf. Proc. 1628, 1003-1008 (2014); doi: 10.1063/1.4902703 (C) 2014 AIP Publishing LLC 978-0-7354-1265-1/\$30.00 
discrete constrained optimization problem (see [7] for a complete proof and details.)

These methods require $O\left(N^{6}\right)$ operations per evaluation of the collision operator, where $N$ is the total number of velocity grid points in each dimension. While typical 3D convolutions can be computed in $O\left(N^{3} \log N\right)$ operations, the presence of convolution weights in the model requires the full $O\left(N^{6}\right)$ computation of the convolution, except for a few special collision models such as hard spheres in 3D and Maxwell molecules in 2D, which can be done with $O\left(N^{3} \log N\right)$ operations using the Carleman representation of the collision integral $[8,9]$. Work by $\mathrm{Hu}$ and Ying extended this idea to the cubic Quantum Boltzmann collision operator, reducing the computational cost from $O\left(N^{9}\right)$ to $O\left(N^{3} \log N\right)$ for the hard spheres model $[10,11]$. The main idea behind these speedups is to form a decomposition of the convolution weights at each quadrature point arising from a discretization of a spherical integral that arises in the model. This work extends that idea to the Boltzmann operator without using the Carleman representation, which restricted the speedup to the collision models listed above.

This article is organized as follows. Firstly we present a review of the original spectral method. Then we present the new decomposition of the convolution weights that allows for fast methods to be used for the convolutions. Finally we present some preliminary numerical results, and conclusions and outlook.

\section{REVIEW OF ORIGINAL NUMERICAL METHOD}

The space homogeneous elastic Boltzmann equation is given by the initial value problem

$$
\frac{\partial}{\partial t} f(\mathbf{v}, t)=Q(f, f)(\mathbf{v}, t)
$$

with

$$
\mathbf{v} \in \mathbb{R}^{d}, \quad f(v, 0)=f_{0}(\mathbf{v}),
$$

where $f(\mathbf{v}, t)$ is a probability density distribution in $\mathbf{v}$-space and $f_{0}$ is assumed to be at least locally integrable with respect to $\mathbf{v}$.

The collision operator $Q(f, f)$ is a bilinear integral form in $(\mathbf{v}, t)$ given by

$$
Q(f, f)(\mathbf{v}, t)=\int_{\mathbf{v}_{*} \in \mathbb{R}^{d}} \int_{\omega \in S^{d-1}} B\left(\left|\mathbf{v}-\mathbf{v}_{*}\right|, \cos \theta\right)\left(f\left(\mathbf{v}_{*}^{\prime}\right) f\left(\mathbf{v}^{\prime}\right)-f\left(\mathbf{v}_{*}\right) f(\mathbf{v})\right) d \omega d \mathbf{v}_{*},
$$

where the velocities $\mathbf{v}^{\prime}, \mathbf{v}_{*}^{\prime}$ are determined through a given collision rule depending on $\mathbf{v}, \mathbf{v}_{*}$. The positive term of the integral in (2) evaluates $f$ in the pre-collisional velocities that can result in a post-collisional velocity the direction $\mathbf{v}$. The scattering cross section $B\left(\left|\mathbf{v}-\mathbf{v}_{*}\right|, \cos \theta\right)$ is a given non-negative function depending on the size of the relative velocity $\mathbf{u}:=\mathbf{v}-\mathbf{v}_{*}$ and $\cos \theta=\frac{\mathbf{u} \cdot \boldsymbol{\omega}}{|\mathbf{u}|}$, where $\omega$ in the $n-1$ dimensional sphere $S^{n-1}$ is referred to as the scattering direction, which coincides with the direction of the post-collisional elastic relative velocity.

The elastic interaction law written in the scattering direction $\omega$ is given by

$$
\begin{aligned}
& \mathbf{v}^{\prime}=\mathbf{v}+\frac{1}{2}(|\mathbf{u}| \omega-\mathbf{u}), \quad \mathbf{v}_{*}^{\prime}=\mathbf{v}_{*}-\frac{1}{2}(|\mathbf{u}| \omega-\mathbf{u}), \\
& B(|\mathbf{u}|, \cos \theta)=|\mathbf{u}|^{\lambda} b(\cos \theta) .
\end{aligned}
$$

The angular cross section function $b(\cos \theta)$ may or may not be integrable with respect to $\theta$; the case when integrability holds is referred to as the Grad cut-off assumption on the angular cross section.

The parameter $\lambda$ regulates the collision frequency as a function of the relative velocity $|\mathbf{u}|$. This parameter corresponds to the interparticle potentials used in the derivation of the collisional cross section and choices of $\lambda$ are denoted as variable hard potentials (VHP) for $0<\lambda<1$, hard spheres (HS) for $\lambda=1$, Maxwell molecules (MM) for $\lambda=0$, and variable soft potentials (VSP) for $-3<\lambda<0$. The $\lambda=-3$ case corresponds to a Coulombic interaction potential between particles. If $b(\cos \theta)$ is independent of $\theta$ we call the interactions isotropic, e.g., in the case of hard spheres in three dimensions. We will only consider isotropic interactions in this paper. 
The key step our formulation of the spectral numerical method is the use of the weak form of the Boltzmann collision operator. For a suitably smooth test function $\phi(\mathbf{v})$ the weak form of the collision integral is given by

$$
\int_{\mathbb{R}^{d}} Q(f, f) \phi(\mathbf{v}) d \mathbf{v}=\int_{\mathbb{R}^{d} \times \mathbb{R}^{d} \times S^{d-1}} f(\mathbf{v}) f\left(\mathbf{v}_{*}\right) B(|\mathbf{u}|, \cos \theta)\left(\phi\left(\mathbf{v}^{\prime}\right)-\phi(\mathbf{v})\right) d \omega d \mathbf{v}_{*} d \mathbf{v} .
$$

If one chooses

$$
\phi(\mathbf{v})=e^{-i \zeta \cdot \mathbf{v}} /(\sqrt{2 \pi})^{d},
$$

then (4) is the Fourier transform of the collision integral with Fourier variable $\zeta$ :

$$
\begin{aligned}
\widehat{Q}(\zeta) & =\frac{1}{(\sqrt{2 \pi})^{d}} \int_{\mathbb{R}^{d}} Q(f, f) e^{-i \zeta \cdot \mathbf{v}} d \mathbf{v} \\
& =\int_{\mathbb{R}^{d} \times \mathbb{R}^{d} \times S^{d-1}} f(\mathbf{v}) f\left(\mathbf{v}_{*}\right) \frac{B(|\mathbf{u}|, \cos \theta)}{(\sqrt{2 \pi})^{d}}\left(e^{-i \zeta \cdot \mathbf{v}^{\prime}}-e^{-i \zeta \cdot \mathbf{v}}\right) d \omega d \mathbf{v}_{*} d \mathbf{v} \\
& =\int_{\mathbb{R}^{d}} G(\mathbf{u}, \zeta) \mathscr{F}[f(\mathbf{v}) f(\mathbf{v}-\mathbf{u})](\zeta) d \mathbf{u},
\end{aligned}
$$

where $\widehat{[\cdot]}=\mathscr{F}(\cdot)$ denotes the Fourier transform and

$$
G(\mathbf{u}, \zeta)=|\mathbf{u}|^{\lambda} \int_{S^{d-1}} b(\cos \theta)\left(e^{-i \frac{\beta}{2} \zeta \cdot|\mathbf{u}| \omega} e^{i \frac{\beta}{2} \zeta \cdot \mathbf{u}}-1\right) d \omega .
$$

Further simplification can be made by writing the Fourier transform inside the integral as a convolution of Fourier transforms:

$$
\widehat{Q}(\zeta)=\int_{\mathbb{R}^{d}} \widehat{G}(\xi, \zeta) \widehat{f}(\zeta-\xi) \widehat{f}(\xi) d \xi
$$

where the convolution weights $\widehat{G}(\xi, \zeta)$ are given by

$$
\widehat{G}(\xi, \zeta)=\frac{1}{(\sqrt{2 \pi})^{d}} \int_{\mathbb{R}^{d}} G(\mathbf{u}, \zeta) e^{-i \xi \cdot u} d \mathbf{u} .
$$

These convolution weights can be precomputed once to high accuracy and stored for future use. For many collision types the complexity of the integrals in the weight functions can be reduced dramatically through analytical techniques.

The computational cost to compute $\widehat{Q}(\zeta)$ is $O\left(N^{6}\right)$, as each of the $N^{3}$ points on the $\zeta$ grid requires computation of a 3D integral. These integrals are highly independent and very amenable to high performance distributed computing, however the major speedbump for modern supercomputers is memory, not computation. There are $N^{6}$ convolution weights to store, which requires, for example $32 \mathrm{~GB}$ of space for $N=40$. Instead we seek a decomposition of $\widehat{G}(\xi, \zeta)$ in a more compact and computationally convenient form.

\section{FORMULATION OF FAST NUMERICAL METHOD}

Our goal is to find a decomposition of $\widehat{G}(\xi, \zeta)$ in the following form:

$$
\widehat{G}(\xi, \zeta)=\sum_{t} \alpha_{t}(\xi) \beta_{t}(\zeta)
$$

then equation (7) can be written as

$$
\begin{aligned}
\widehat{Q}(\zeta) & =\sum_{t} \int_{\mathbb{R}^{d}} \alpha_{t}(\xi) \beta_{t}(\zeta) \hat{f}(\zeta-\xi) \hat{f}(\xi) d \xi \\
& =\sum_{t} \beta_{t}(\zeta) \int_{\mathbb{R}^{d}} \hat{f}(\zeta-\xi)\left[\alpha_{t}(\xi) \hat{f}(\xi)\right] d \xi
\end{aligned}
$$


which is now a pure convolution, and can be sped up by the Fast Fourier Transform.

Recall from above that

$$
\widehat{G}(\xi, \zeta)=\frac{1}{(\sqrt{2 \pi})^{3}} \int_{\mathbb{R}^{3}}|u|^{\lambda} \int_{\mathbb{S}^{2}} b\left[e^{-i \frac{1}{2}|u| \zeta \cdot \omega+i\left(\frac{1}{2} \zeta-\xi\right) \cdot u}-e^{-i \xi \cdot u}\right] d \omega d u .
$$

We integrate $u$ first in the space where $f$ lives in. Let

$$
u=r \omega_{\theta, \varphi}, \quad \omega_{\theta, \varphi}=(\cos \theta \sin \varphi, \sin \theta \sin \varphi, \cos \varphi), \quad d u=r^{2} \sin \varphi d r d \varphi d \theta,
$$

then

$$
\widehat{G}(\xi, \zeta)=\frac{1}{(\sqrt{2 \pi})^{3}} \int_{0}^{2 \pi} \int_{0}^{\pi} \int_{0}^{R} r^{\lambda+2} \sin \varphi \int_{\mathbb{S}^{2}} b\left[e^{-i \frac{r}{2} \zeta \cdot \omega+i r\left(\frac{1}{2} \zeta-\xi\right) \cdot \omega_{\theta, \varphi}}-e^{-i r \xi \cdot \omega_{\theta, \varphi}}\right] d \omega d r d \varphi d \theta
$$

We next numerically integrate $r, \theta$, and $\varphi$,

$$
\widehat{G}(\xi, \zeta)=\frac{1}{(\sqrt{2 \pi})^{3}} \sum_{r, \theta, \varphi} q_{r} q_{\theta} q_{\varphi} r^{\lambda+2} \sin \varphi \int_{\mathbb{S}^{2}} b\left[e^{-i \frac{r}{2} \zeta \cdot \omega+i r\left(\frac{1}{2} \zeta-\xi\right) \cdot \omega_{\theta, \varphi}}-e^{-i r \xi \cdot \omega_{\theta, \varphi}}\right] d \omega
$$

where $q_{r}, q_{\theta}$, and $q_{\varphi}$ are the quadrature weights. The integral part in the above equation is given by

$$
\begin{aligned}
& \int_{\mathbb{S}^{2}} b\left[e^{-i \frac{r}{2} \zeta \cdot \omega+i r\left(\frac{1}{2} \zeta-\xi\right) \cdot \omega_{\theta, \varphi}}-e^{-i r \xi \cdot \omega_{\theta, \varphi}}\right] d \omega \\
= & e^{-i r \xi \cdot \omega_{\theta, \varphi}} e^{i \frac{1}{2} r \zeta \cdot \omega_{\theta, \varphi}} \int_{\mathbb{S}^{2}} b e^{-i \frac{r}{2} \zeta \cdot \omega} d \omega-e^{-i r \xi \cdot \omega_{\theta, \varphi}} \int_{\mathbb{S}^{2}} b d \omega \\
= & A_{r, \theta, \varphi}(\xi)\left(B_{r, \theta, \varphi}(\zeta)-C_{\theta, \varphi}\right),
\end{aligned}
$$

where

$$
A_{r, \theta, \varphi}(\xi)=e^{-i r \xi \cdot \omega_{\theta, \varphi}}, \quad B_{r, \theta, \varphi}(\zeta)=e^{i \frac{r}{2} \zeta \cdot \omega_{\theta, \varphi}} \int_{\mathbb{S}^{2}} b e^{-i \frac{1}{2} r \zeta \cdot \omega} d \omega, \quad C_{\theta, \varphi}=\int_{\mathbb{S}^{2}} b d \omega
$$

Function $A$ is readily known. Functions $B$ and $C$ involving integrals in $\omega$ can be precomputed and tabulated. In particular, if $b \equiv 1 /(4 \pi)$, then

$$
B(\zeta)=e^{i \frac{1}{2} r \zeta \cdot \omega_{\theta, \varphi}} \operatorname{sinc}\left(\frac{r|\zeta|}{2}\right), \quad C \equiv 1
$$

Inserting expansion (14) into (13), we obtain

$$
\widehat{G}(\xi, \zeta)=\frac{1}{(\sqrt{2 \pi})^{3}} \sum_{r, \theta, \varphi} q_{r} q_{\theta} q_{\varphi} r^{\lambda+2} \sin \varphi A_{r, \theta, \varphi}(\xi)\left[B_{r, \theta, \varphi}(\zeta)-C_{\theta, \varphi}\right]
$$

which is exactly the type of decomposition we wanted at the beginning.

The final cost for computing $\widehat{Q}(\zeta)$ would be $O\left(M_{r} M_{\theta} M_{\varphi} N^{3} \log N\right)$. From experience $M_{\theta}, M_{\varphi}$ can be chosen relatively small and $M_{r}$ should be $O(N)$, the cost is roughly $O\left(M^{2} N^{4} \log N\right)$. The storage requirement for precomputation is $O\left(M_{r} M_{\theta} M_{\varphi} N^{3}\right)$, which is roughly $O\left(M^{2} N^{4}\right)$. As reference, the cost and storage of original method are both $O\left(N^{6}\right)$.

\section{NUMERICAL RESULTS}

To verify the numerical method, we use the Bobylev-Krook-Wu solution to the homogeneous Boltzmann equation. This is an explicit solution that takes the form

$$
f(\mathbf{v}, t)=\frac{1}{2(2 \pi K)^{3 / 2}}\left(\frac{5 K-3}{K}+\frac{1-K}{K^{2}}|\mathbf{v}|^{2}\right) e^{-|\mathbf{v}|^{2} / 2 K},
$$




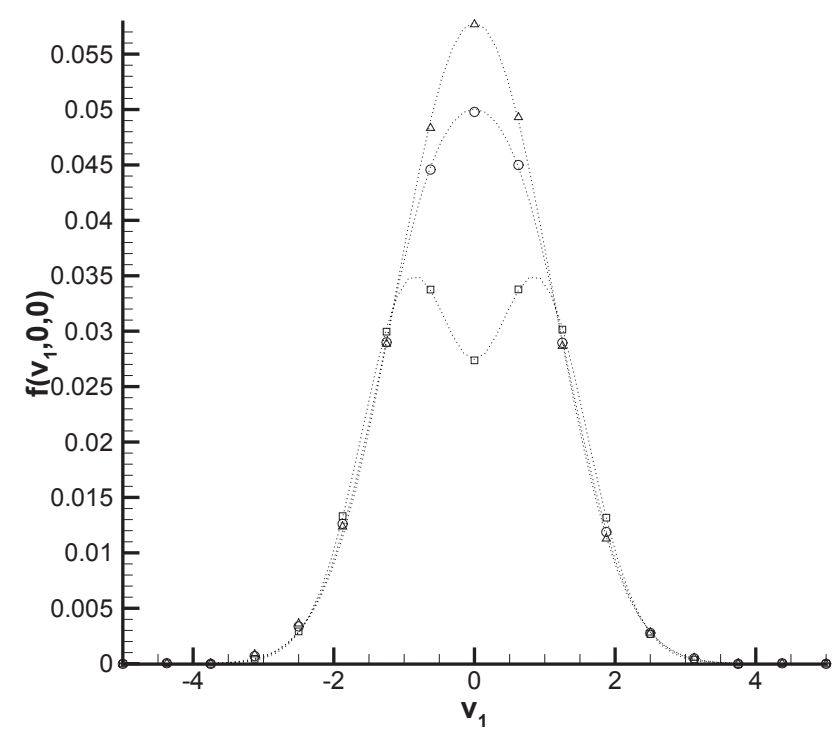

FIGURE 1. Slice of Bobylev-Krook-Wu soluition with $N=17, M_{\theta}=M_{\phi}=8$. Squares: solution at $t=6.5$; circles: solution at $t=8.5$; triangles: solution at $t=10.5$. Dotted lines: true solution.

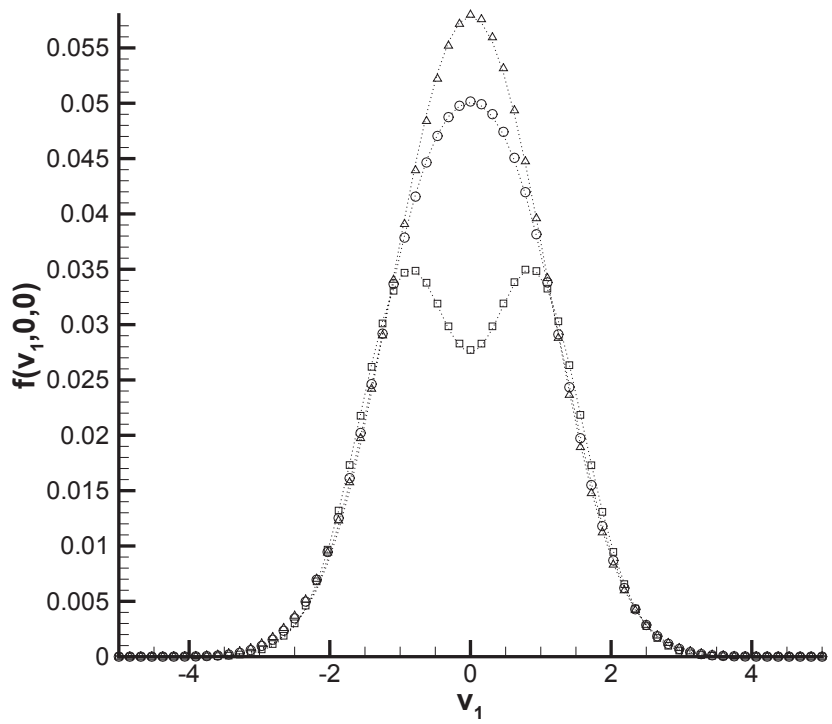

FIGURE 2. Slice of Bobylev-Krook-Wu soluition with $N=65, M_{\theta}=M_{\phi}=8$. Squares: solution at $t=6.5$; circles: solution at $t=8.5$; triangles: solution at $t=10.5$. Dotted lines: true solution.

where $K(t)=1-e^{-t / 6}$. We take the initial time $t_{0}=5.5$ to ensure the distribution is nonnegative.

In Fig. 1 we show the slice of the distribution $f\left(v_{1}, 0,0\right)$ computed with $N=17, M_{\theta}=M_{\phi}=8$ (symbols) against the exact solution (dashed lines) at times $t=6.5,8.5,10.5$, which shows good agreement. In Fig. 2, we show the same computation with $N=65, M_{\theta}=M_{\phi}=8$, which also shows good agreement with the true solution despite the large 
TABLE 1. Time to evaluate collision operator. $M_{\theta}=M_{\phi}=8$ for fast method. Entries with an asterix required more memory than was available on the compute node; dummy values are used for the weights instead in order to estimate the computation time.

\begin{tabular}{cccccccc}
\hline$N$ & 17 & 25 & 33 & 41 & 49 & 57 & 65 \\
Original & 0.06 & 0.40 & 2.10 & $6.44 *$ & $18.75^{*}$ & $45.94 *$ & $100.11 *$ \\
Fast method & 0.39 & 0.984 & 2.653 & 7.90 & 8.57 & 17.76 & 22.73 \\
\hline
\end{tabular}

difference between $M$ and $N$.

To check the computational efficiency of the method, we compare the fast method described in the third section to the original spectral method described in the second section. In table 1, we show a comparison of the time required for a single evaulation of the collision operator. These computations were performed on the Stampede supercomputer at the Texas Advanced Computing Center. Entries marked by an asterisk require too much storage for the convolution weights to fit in the memoty of a single node, and are instead computed using stored dummy values.

\section{CONCLUSIONS AND OUTLOOK}

As can be seen from the computational time scaling results, this method offers the ability to move past the memory bottlenecks required to compute problems that require large values of $N$. While it is still expensive per evaluation, it is now computationally feasible. However for relatively small values of $N$ the original method outperforms the fast method due to the lack of overhead caused by computing all of the extra FFTs. It only requires a single FFT and IFFT and a simple update to $\widehat{Q}$ when computing the convolution, as opposed to the $N M^{2}$ FFTs and additional quadrature-related multiplications required for the fast method.

Future work will seek to further demonstrate the utility of this method by computing problems that require a high amount of resolution, such as high Mach number shocks and bump on tail problems. We will also extend it to the case of angularly dependent cross sections, which requires a more careful handling of the weight decomposition especially in the case of grazing collisions [12]. We will also examine the role of further leveraging high performance computing resources to further decrease the computational requirements of the fast method.

\section{ACKNOWLEDGMENTS}

This work has been supported by the NSF under grants DMS-0636586, DMS-1109625, and NSF RNMS (KI-Net) grant \#11-07465. Computations were performed using Stampede at the Texas Advanced Computing Center (TACC), an NSF XSEDE resource.

\section{REFERENCES}

1. A. V. Bobylev, Mathematical Physics Reviews 7, 111-233 (1988), Soviet Sci. Rev. Sect. C Math. Phys.Rev., 7, Harwood Academic Publ., Chur.

2. L. Pareschi, and B. Perthame, Trans. Theo. Stat. Phys. 25, 369-382 (1996).

3. L. Pareschi, and G. Russo, SIAM J. Num. Anal. pp. 1217-1245 (2000).

4. I. Ibragimov, and S. Rjasanow, Computing 69, 163-186 (2002).

5. I. M. Gamba, and S. H. Tharkabhushanam, J. Comput. Phys 228, 2016-2036 (2009).

6. I. M. Gamba, and S. H. Tharkabhushanam, J. Comput. Math 28, 430-460 (2010).

7. R. Alonso, I. M. Gamba, and S. H. Tharkabhushanam, Accuracy and consistency of Lagrangian based conservative spectral method for space-homogeneous Boltzmann equation (2014), submitted.

8. F. Filbet, C. Mouhot, and L. Pareschi, SIAM J. Sci. Comput. 28, 1029-1053 (2006).

9. C. Mouhot, and L. Pareschi, Math. Comput. 75, 1833-1852 (2006).

10. J. Hu, and L. Ying, Commun. Math. Sci. 10, 989-999 (2012).

11. J. Hu, and L. Ying, A fast algorithm for the energy space boson boltzmann collision operator (2014), to appear.

12. I. M. Gamba, and J. Haack, J. Comput. Phys. 270, 40-57 (2014). 